\title{
Registro CENIC-SBHCI: Envidar Novas Ideias para Renovar um Projeto Perene
}

\author{
Ver artigo relacionado \\ na página 131
}

\author{
Luiz Alberto Mattos ${ }^{1}$
}

$\mathbf{N}$ esta edição da Revista Brasileira de Cardiologia Invasiva, Matte et al. ${ }^{1}$ apresentam uma renovação dos dados demográficos e resultados imediatos observados no último quinquênio, no Brasil, após a aplicação da intervenção coronária percutânea (ICP) primária na vigência das primeiras horas após o estabelecimento de infarto agudo do miocárdio (IAM) com evidência de supradesnivelamento do segmento ST.

Essas informações foram retiradas do registro pertencente à Sociedade Brasileira de Hemodinâmica e Cardiologia Intervencionista (SBHCI), CENIC (Central Nacional de Intervenções Cardiovasculares), registro esse construído por meio da contribuição espontânea dos associados dessa agremiação, os cardiologistas intervencionistas.

O relato atual é primoroso em detalhamento, extraindo todas as informações possíveis reunidas na ficha de coleta de dados, permitindo aferir a evolução numérica da aplicação desse método de reperfusão coronária assim como seu efeito na redução da morbidade e mortalidade dessa que é a principal manifestação e revestida de maior gravidade clínica da doença arterial coronária.

Mas será possível exarar conclusões com o nível de consistência que os autores subscrevem ao final da referida pesquisa? Uma revisão do passado sempre promove reflexões para aprimoramentos no futuro e a possível resposta a esse questionamento.

O registro CENIC foi idealizado na administração da SBHCl iniciada em 1991, na época ainda um departamento da Sociedade Brasileira de Cardiologia (SBC), presidida naquele biênio (1991-1992) pelo Dr. José Nogueira Paes (Fortaleza, CE, Brasil). Essa iniciativa pioneira, e que merece menção de louvor, catalisava um desejo e uma necessidade societária premente de obter conhecimento da prática clínica intervencionista no Brasil, notadamente, da efetivação da angioplastia coronária, hoje ICP, relacionada a indiscutível liderança na prevalência da doença arterial coronária.

Na oportunidade, o Dr. Nogueira Paes convidou a Profa. Dra. Amanda G. M. R. Sousa (São Paulo, SP, Brasil) para gerenciar o futuro registro. Assim, sob a batuta dessa maestrina única da cardiologia intervencionista brasileira foi gestado o registro CENIC, do marco zero. Por pouco mais de uma década (1991-2002), esse registro esteve sob seu gerenciamento, e tive então a honrosa oportunidade de, atendendo a seu convite, auxiliá-la diariamente na construção, direção e controle do mesmo. O departamento não ofertava uma sede própria, sendo abrigado, inicialmente, no Instituto Dante Pazzanese de Cardiologia (São Paulo, SP, Brasil). Importante recordar que, nesses anos, a informática engatinhava e era restrita a muito poucos, e uma rede federativa necessita ser tecida, para que, mediante a união e o compromisso de todos, lográssemos o êxito e o objetivo almejados. Um ano foi despendido na preparação da logística e na contratação de colaboradores dedicados, e em 1992 iniciava-se a coleta de dados.

Em 1994, portanto menos de dois anos após sua criação, publica-se, nos Arquivos Brasileiros de Cardiologia, o primeiro relatório desse registro, compreendendo o biênio 1992-1993 (16.429 pacientes analisados), amálgama consistente da contribuição de 97 sócios, aderência inicial próxima de $80 \% .^{2}$

Desde então, a CENIC somente floresceu, refletindo o crescimento vertiginoso da prática da ICP no Brasil, com número crescente de contribuintes, de menos de uma centena em 1994 para próximo de 300, como evidenciado em mais esse relato de Matte et al. ${ }^{1}$. As publicações científicas se sucederam, se multiplicaram, diversificaram as variáveis analisadas, e têm sido presença constante, seja no órgão de divulgação científica da $\mathrm{SBHCl}$ como da SBC, assim como em apresentações no exterior, notadamente nos congressos norte-americanos de car-

\footnotetext{
1 Instituto Dante Pazzanese de Cardiologia - São Paulo, SP, Brasil. Correspondência: Luiz Alberto Mattos. Av. Jandira, 550/121 - Indianópolis - São Paulo, SP, Brasil - CEP 04080-003 E-mail: pivmattos@uol.com.br 
diologia clínica. ${ }^{3-10} \mathrm{O}$ registro CENIC se constitui em um dos pilares societários, motivo de orgulho dos sócios, fonte de consulta e informação constante, fomento à discussão de seus resultados periódicos, nos diversos fóruns em que se necessita apresentar dados brasileiros intervencionistas.

Porém, o crescimento geométrico promove novos desafios e penso que essa análise de Matte et al. ${ }^{1}$ é um aríete para que uma renovação seja efetivada, de fato, no registro. A manutenção de um registro de modo aberto, com temporalidade infinita, é uma contenda tremenda, contenda essa relacionada à manutenção da aderência dos sócios contribuintes. Em tempo, o registro CENIC carrega em sua certidão de nascimento três características que justamente são os maiores desafios à manutenção de seu brilho, quais sejam: é uma contribuição espontânea e não compulsória, é de preenchimento exclusivo do investigador sem adjudicação dos dados, e compreende um período curto de análise, hospitalar e, com muito esforço, até o final dos primeiros 30 dias após a realização da ICP. ${ }^{1-10}$

Uma análise comparativa do quatriênio 1997-20004 com a apresentação deste quinquênio recente ${ }^{1}$ confrontada com a publicação dos resultados observados em pacientes submetidos a ICP primária sob a égide do Sistema Único de Saúde (SUS) do Brasil (Ministério da Saúde) ${ }^{11}$ suscita a evidência da discordância marcante de resultados (Tabela). Se por um lado observa-se o dobro da efetivação de procedimentos em infartados, ao longo dos anos denota-se, contudo, discordância no marcante número de notificações (55\% menor; CENIC 2006-2010 vs. SUS 2005-2008) e, principalmente, em mortalidades muito diversas.

O cotejamento do relato de Matte et al. ${ }^{1}$ com a recente contribuição de Piegas e Haddad ${ }^{11}$ expõe as fragilidades do nascedouro do registro CENIC, quais sejam a baixa aderência à notificação (método facultativo) e a ausência de adjudicação e revisão independente dos dados enviados à CENIC. Muito provavelmente, essa taxa muito reduzida de mortalidade hospitalar, exposta pelos autores, em período temporal que se sobrepõe ao relato do SUS, reflete, novamente, a não comunicação de eventos fatais subsequentes ao final do procedimento ou após as primeiras 24 horas, retirando assim a oportunidade de fornecer conclusões substanciosas oriundas desse relato.

Ao questionamento inicial, eu responderia que o relato atual, a despeito do esmero dedicado e meticuloso dos autores na sua apresentação, não representa a realidade da prática clínica vigente da ICP efetivada em pacientes infartados no Brasil, seja numericamente seja na ocorrência dos desfechos graves.

Esse relato estimula sim que envidemos esforços concentrados, despejando novas ideias para que sigamos com o registro CENIC, perene que é, transparente, robusto, consistente, engalanando novamente esse que é um dos pilares da $\mathrm{SBHCl}$.

Uma nova administração se iniciará muito em breve (2012-2013) e chega o momento de executarmos um plano de ação de revitalização da CENIC. As administrações passadas e a atual seguiram investindo nesse registro, adotando moderno sistema de coleta eletrônico, cauterizando diferenças de captura de dados do passado, ampliando o escopo de dados coletados, esforços esses, contudo, insuficientes para retornar esse apontamento ao pináculo que merece e ao que desejamos.

Minha recomendação, de modo sucinto, para que seja possível obter novamente uma fotografia com nitidez cristalina da prática clínica brasileira intervencionista dedicada à ICP e ao tratamento da doença arterial coronária, é que medidas de impacto sejam efetivadas para o resgate desse projeto. O registro CENIC pode continuar aberto e infinito na captura de dados, para todos, porém, mediante a criação de novas variações de pesquisa, se faz necessário obter a coleta de dados em períodos determinados, reunindo centros interessados e comprometidos com o envio de dados, de modo federativo e contínuo, no período estipulado, associado à adjudicação de dados de maneira independente, pelo método de amostragem, com o amparo de monitores e colaboradores exclusivos para tal tarefa. Encontros periódicos ou virtuais se fazem necessários para manter

TABELA 1

Resultado comparativo da efetivação da intervenção coronária percutânea na vigência de infarto agudo do miocárdio em registros brasileiros

\begin{tabular}{lccr}
\hline Registro & CENIC & CENIC $^{\mathbf{1}}$ & SUS $^{11}$ \\
\hline Período & $1997-2000$ & $2006-2010$ & $2005-2008$ \\
Método para notificação & Facultativo & Facultativo & Compulsório \\
ICPs no IAM, pacientes & 9.371 & 20.004 & 33.286 \\
Média anual, pacientes & 2.342 & 4.000 & 8.321 \\
Mortalidade hospitalar, \% & 5,75 & 2,8 & 6,35 \\
\hline
\end{tabular}

CENIC = Central Nacional de Intervenções Cardiovasculares; ICPs = intervenções coronárias percutâneas; IAM = infarto agudo do miocárdio; SUS = Sistema Único de Saúde. 
o grupo selecionado, agregado, ativo e estimulado e assim estender, nesse novo projeto, a coleta de dados para um seguimento clínico no mínimo de 12 meses. Associado a essas medidas, as informações obtidas devem ser de propriedade exclusiva e impenetrável da agremiação e de seus sócios contribuintes, mediante o retorno ao gerenciamento próprio dos dados, condição esta inicialmente existente, porém modificada em administração passada.

Um dito popular deve emoldurar esse novo desafio societário, qual seja, "informação verdadeira é poder", e, portanto, há que se renovar as forças de nosso registro para que poderoso novamente se torne, e, por conseguinte, nossa $\mathrm{SBHCl}$.

\section{CONFLITO DE INTERESSES}

O autor declara não haver conflito de interesses relacionado a este manuscrito.

\section{REFERÊNCIAS}

1. Matte BS, Bergoli LCC, Balvedi JA, Zago AC. Perfil da intervenção coronária percutânea no infarto agudo do miocárdio com supradesnivelamento do segmento ST no Brasil de 2006 a 2010: Registro CENIC. Rev Bras Cardiol Invasiva. 2011;19(2):131-7.

2. Sousa AGMR. Percutaneous cardiovascular intervention procedures in Brazil (1992-1993): report of the National RegistryNational Center for Cardiovascular Interventions. Arq Bras Cardiol. 1994;62(4):217-23.

3. Sousa AGMR, Mattos LA, Campos Neto CM, Carvalho HG, Stella FP, Nunes G. Percutaneous myocardial revascularization procedures in Brazil in 1996-1997 compared to the 1992-1993 period: a report of the National Registry - Na- tional Center for Cardiovascular Interventions (CENIC). Arq Bras Cardiol. 1998;70(6):423-30.

4. Mattos LA, Sousa AG, Pinto IM, Silva ER, Carneiro JK, Sousa $\mathrm{JE}$, et al. A comparison of rescue and primary percutaneous coronary interventions for acute myocardial infarction: a multicenter registry report of 9,371 patients. Arq Bras Cardiol. 2004;82(5):440-4

5. Mattos LA, Sousa A, Campos Neto C, Labrunie A, Alves CR, Saad J. Primary coronary angioplasty and stent implantation in acute myocardial infarction: comparative analysis of the inhospital results in the CENIC/SBHCI Registry. Arq Bras Cardiol. 1999;73(6):475-84.

6. Mattos LA, Sousa AG, Campos Neto CM, Labrunie A, Alves $C R$, Feres $F$, et al. The use of primary stenting or balloon percutaneous transluminal coronary angioplasty for the treatment of acutely occluded saphenous vein grafts: results from the Brazilian National Registry-CENIC. Central Nacional de Intervenções Cardiovasculares. Arq Bras Cardiol. 2001;76(6): 483-95.

7. Mattos LA, Sousa AGMR, Pinto IMF, Campos Neto CM, Labrunie A, Alves CR, et al. Primary coronary angioplasty in 9,434 patients during acute myocardial infarction: predictors of major in-hospital adverse events from 1996 to 2000 in Brazil. Arq Bras Cardiol. 2002;79(4):412-8.

8. Moscoso I, Garcia LC, Dourado GO, Mauro MF, Caramori P, Coelho W, et al. Influence of Diabetes Mellitus on immediate results of coronary stent: National Center for Cardiovascular Interventions (CENIC) data analysis. Arq Bras Cardiol. 2006; 86(3):181-90.

9. Cardoso CO, Quadros AS, Mattos LA, Gottschall CA, SarmentoLeite RE, Marin-Neto JA. Use of drug-eluting stents in Brazil: the CENIC (National Registry of Cardiovascular Interventions) registry. Arq Bras Cardiol. 2007;89(6):356-61.

10. Andrade PE, Tebet MA, Andrade MVA, Labrunie A, Mattos LA. Radial approach in percutaneous coronary interventions: current status in Brazil. Arq Bras Cardiol. 2011;96(4):312-6.

11. Piegas LS, Haddad N. Percutaneous coronary intervention in Brazil: results from the Brazilian Public Health System. Arq Bras Cardiol. 2011;96(4):317-24. 